\title{
A Geometric Programming Solution to an Economic Production Quantity Model with Ranking Fuzzy Numbers Cost Parameters
}

\author{
K. Jayanthi, W. Ritha
}

\begin{abstract}
-on this paper, a financial creation quantity is figured to hold up a era framework with restrict, adaptability, unwavering first-class, and circumstance supportability with switch fee of non-appealing object. The cost parameters are spoken to as a trapezoidal fluffy numbers and those fluffy numbers are communicated as positioning fluffy numbers with satisfactory guess period in-between. Geometric programming method is attached to determine the precise selections in close from. A numerical model is given to help the difficulty.
\end{abstract}

Keywords: Optimal production quantity, Ranking fuzzy number, Best interval approximation for trapezoidal number, Geometric programming technique.

\section{INTRODUCTION}

All matters considered, it isn't always continuously practicable to get the ideal statistics approximately stock parameters. This sort of free information isn't always in every case all round spoken to by ordinary elements 18 Nirmal Kumar Mandal selected from danger conveyance. So primary leadership techniques underneath vulnerability are required. to manipulate this vulnerability and unsure statistics, the idea of fluffiness may be connected. The stock rate parameters, as an example, request, retaining fee, creation charge, switch value are idea to be adaptable as an example fluffy in nature. those parameters may be spoken to by means of fluffy numbers. a talented method for raking fluffy numbers has a fantastic system to address the fluffy numbers in a fluffy primary management trouble. Hariri and Abou-el-ata [6], Abou-el-ata and kotb [7], Abou-el-ata et al. [8], built up some inventory models with variable inventory expenses and settled them via GP approach.

Duffin et al. [16] first verified that the GP approach can be implemented woth a few factors of interest for improvement troubles of a selected sort. Later $[1,6,7,17,18]$ tackled some stock troubles utilising GP strategy. Cao $[19,20]$ furnished fluffy geometric programming which end up later used by precise analysts in searching after fluffy fundamental control troubles. Roy and Maiti [25] unraveled the vintage style EOQ model for a solitary difficulty in fluffy situation. Roy and Maiti [26] additionally tested the fluffy EOQ model with

Revised Manuscript Received on July 10, 2019.

K. Jayanthi, Department of Mathematics, Holy Cross College (Autonomous), Trichirapalli, Tamil Nadu, India.

W. Ritha, Department of Mathematics, Holy Cross College (Autonomous), Trichirapalli, Tamil Nadu, India. interest subordinate unit price and uncertain stockpiling region via manner of every fluffy GP and NLP method. Mandal et al. [27] pointed out a multi-issue fluffy stock trouble with 3 limitations and unraveled with the aid of GP device. Mandal and Roy [28] planned a inventory model with inventory ward inventory fees that they have got understood through the use of GP system.

The corporation goes for carrying out maximum intense gain which requires nonstop execution of the creation manner, however certain limitations like device breakdown, deficiency of substances, absence of proficient and semi-gifted specialists exist which is probably overwhelmed by using keeping up a top notch re-appropriating in desire to acquisition. along these strains to maintain up a advent framework with limit, adaptability, unwavering great and situation manageability, we've got precise a financial generation quantity of the framework.

\section{MATHEMATICAL MODEL:}

\subsection{Assumptions:}

1. The rate of production and demand remains constant.

2. Continuous replenishment takes place.

3. Deficit and surplus of stock do not take place.

4. The cost of technology is incurred to maintain the standard of production system.

\subsection{Notations:}

A Fixed set up cost

$\mathrm{p}$ production rate

D Demand rate

c unit production cost

$\mathrm{h}$ holding cost per unit of time

B Cost of technology, resource and design

E Extra expenditure for cleaner technologies at times of depreciation of machinery

$\mathrm{K}$ The expenditure to overcome the limitation of different complexities to increase the reliability.

$\mathrm{x} \mathrm{D} / \mathrm{P}$

$1-\mathrm{x}$ the fraction of time the production process

Mi materials which does not enter the production process $(\mathrm{i}=1,2,3 \ldots \ldots)$

Cicost of materials Mi

$\mathrm{d}$ disposal cost of non marketable product.

$\mathrm{T}$ Processing cost of non product output 


\section{A GEOMETRIC PROGRAMMING SOLUTION TO AN ECONOMIC PRODUCTION QUANTITY MODEL WITH RANKING FUZZY NUMBERS COST PARAMETERS}

$\mathrm{O}$ cost of incurring the outsourcing

\section{CRISP MODEL}

consider a era area which makes single object. The contribution to the creation framework is the crude materials, but not every one of the materials enter the generation technique. finally the age of waste (non-appealing item) takes place at this component which should be taken consideration off. As in in recent times the organizations stroll closer to the shared fear of nature, switch fee is received for such non attractive object honestly as non-object yield (the waste that is created towards the end of the era). The goes for attaining greatest advantage which requires constant execution of the era technique, but at the equal time wonderful limitations like system breakdown, deficiency of substances, absence of gifted experts exist which may be beaten with the useful resource of preserving up a genuinely perfect redistributing in desire to obtainment. on this manner to preserve up a generation framework with restrict, adaptability, dependability and situation manageability an monetary creation amount version is exceptional as pursues.

The EPQ cost per unit of time $\mathrm{C}(\mathrm{Q})=\mathrm{AD} / \mathrm{Q}+\mathrm{hQ}(1-\mathrm{x}) / 2$ The production cost per cycle $\mathrm{Cp}(\mathrm{Q})=\mathrm{cQ}$

The costs of materials which do not enter the production process $=\sum_{i=1}^{n} C_{i} M_{i}$

The disposal cost of non-marketable product $=$

The inventory cost per unit of time is given as

$\mathrm{T}(\mathrm{C}(\mathrm{Q}))$

$\frac{A D}{Q}+\frac{h Q(1-x)}{2}+\left[\sum_{i=1}^{n} C_{i} M_{i}+\sum_{i=1}^{n} d M_{i}+c Q+B+E+K+T+o\right] \frac{D}{Q}$

The optimal solution is $\mathrm{Q}=$

$$
\sqrt{\frac{2 D\left[A+\sum_{i=1}^{n} C_{i} M_{i}+\sum_{i=1}^{n} d M_{i}+B+K+E+T+O\right]}{h(1-x)}}
$$

\section{FUZZY MODEL}

Here, D, $\mathrm{C}_{\mathrm{i}}, \mathrm{d}, \mathrm{B}, \mathrm{K}, \mathrm{E}, \mathrm{T}, \mathrm{O}, \mathrm{h}$ are assumed as fuzzy numbers.

The fuzzy inventory total cost per unit of time is given as

$$
\overline{T(C(Q)}=\left[A+\sum_{i=1}^{n} C_{i} M_{i}+\sum_{i=1}^{n} d M_{i}+c Q+B+E+K+T+o\right] \frac{\bar{D}}{Q}+\frac{\bar{h} Q(1-x)}{2}
$$

\section{NEAREST INTERVAL APPROXIMATION}

Suppose $\bar{A}$ is fuzzy number with $\alpha$ cut $\left[\mathrm{A}_{\mathrm{L}}(\alpha), \mathrm{A}_{\mathrm{R}}\right.$ $(\alpha)]$. Then

The interval

$$
C_{d}(\bar{A})=\left[\int_{0}^{1}\left[A_{L}(\alpha) d \alpha, \int_{0}^{1} A_{R}(\alpha) d \alpha\right]\right.
$$

with respect to the metric d. Let $\bar{A}=(\mathrm{a}, \mathrm{b}, \mathrm{c}, \mathrm{d}$,$) be a$ trapezoidal fuzzy number. The $\cdot$ - cut interval of is defined as $\left[A_{L}(\alpha), A_{R}(\alpha)\right]$ where $A_{L}(\alpha)$ a+ (b-a) $\alpha$ and $A_{R}(\alpha)=d-$ (d-c) $\alpha$. By nearest interval approximation method the lower limit of the interval is $\mathrm{C}_{\mathrm{L}}=\frac{a+b}{2}$ and upper limit of the interval is $\mathrm{C}_{\mathrm{R}}=\frac{c+d}{2}$.

\section{RANKING FUZZY NUMBERS OF COST PARAMETERS WITH BEST APPROXIMATION INTERVAL}

The best approximation interval of $\bar{A}$ is $\left[\mathrm{A}_{\mathrm{L}}(\alpha), \mathrm{A}_{\mathrm{R}}(\alpha)\right]$. The ranking fuzzy number of the best approximation interval $\left[A_{L}(\alpha), A_{R}(\alpha)\right]$ is defined as a convex combination of lower and upper boundary of the best approximation interval. Let $\lambda \in[0,1]$ is pre-assigned parameter, called degree of optimism. Therefore, the ranking fuzzy number of $\bar{A}$ is defined by $R_{\lambda, f}(\bar{A})=\lambda A_{R}(\alpha)+(1-\lambda) A_{L}(\alpha)$.

A large value of $\lambda$ specifies the higher degree of optimism. When $\lambda=0, \mathrm{R}(\bar{A})=\mathrm{A}_{\mathrm{L}}(\alpha)$ expresses that the decision maker`s viewpoint is completely pessimistic. When $\lambda=1$, $\mathrm{R}(\bar{A})=\mathrm{A}_{\mathrm{R}}(\alpha)$ expresses that the decision maker`s attitude is completely optimistic. When $\lambda=1 / 2, \mathrm{R}$ $(\bar{A}) 1 / 2,\left(\mathrm{~A}_{\mathrm{L}}(\alpha)+\left(\mathrm{A}_{\mathrm{R}}(\alpha)\right)\right.$ reflects moderately optimistic or neutral attitude of the decision maker.

Ranking fuzzy numbers of $\bar{A}$ is
$R_{\lambda, f}(\bar{A})=\int_{1}^{0}\left[\lambda A_{R}(\alpha)+(1-\lambda) A_{L}(\alpha)\right] f(\alpha) d \alpha / \int_{1}^{0} f(\alpha) d \alpha$

And we let $\mathrm{f}(\alpha)$. If $\bar{A}=(\mathrm{a}, \mathrm{b}, \mathrm{c}, \mathrm{d}$,$) is a trapezoidal fuzzy$ number, then $A_{L}(\alpha)=a+\alpha(b-a)$ and $A_{R}(\alpha)=d-\alpha(d-c)$. The lower limit of the interval is $A_{L}(\alpha)=(a+2 b) / 3$, and the upper limit of the interval is $A_{R}(\alpha)=(2 c+d) / 3$. Then the corresponding ranking fuzzy number is

$$
R_{\lambda, f}(\bar{A})=2 \int_{1}^{0}\left[\lambda\left(\frac{2 c+d}{3}\right)+(1-\lambda)\left(\frac{a+2 b}{3}\right)\right] \alpha d \alpha
$$

$R_{\lambda, f}(\bar{A})=\frac{1}{3} \lambda(2 c+d)+(1-\lambda)(a+2 b)$

\section{GEOMETRIC PROGRAMMING PROBLEM}

Primal problem: Primal Geometric programming (PGP) problem is

Minimize $g_{o}(t) \sum_{K=1}^{T_{o}} C_{O k} \prod_{k=1}^{m} t_{j}^{\alpha_{o l j}}$
Subject to $\sum_{K=1}^{T_{o}} C_{r k} \prod_{j=1}^{m} t_{j}^{\alpha_{r k j}} \leq 1, \quad(\mathrm{r}=1,2, \ldots . . \mathrm{I}), \mathrm{j}=$

$(1,2,3, \ldots . . . m) t_{j}>0$

Where $\mathrm{C}_{0 \mathrm{k}}>0\left(\mathrm{k}=1,2, \ldots \mathrm{T}_{0}\right)$ $\mathrm{C}_{\mathrm{kk}}$ and $\alpha_{\mathrm{rk}}$ are real numbers. It is constrained polynomial

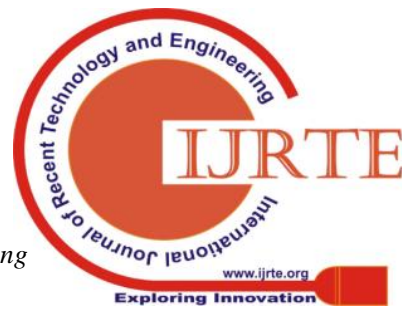


geometric problem. The number of term each polynomial constrained functions varies and it is denoted by $\mathrm{T}$, for each $\mathrm{r}$ $=0,1,2, \ldots .$. Let $\mathrm{T}=\mathrm{T}_{0}+\mathrm{T}_{1}+\mathrm{T}_{2}+\ldots . .+\mathrm{T}_{1}$ be the total number of terms in the primal program. The Degree of difficulty is (DD) $=\mathrm{T}-(\mathrm{m}+1)$

\section{Dual problem:}

Maximize $=\prod_{r=0}^{l} \prod_{k=1}^{T^{r}}\left(\frac{C_{r k}}{\delta_{r k}}\right)^{\delta r k}\left(\sum_{S=1+T_{r+1}}^{T}\left(\delta_{r s}\right)^{\delta r k}\right.$

Subject to $\sum_{k=1}^{T_{0}} \delta_{0 k}=1$ (Normality condition)

$\sum_{r=0}^{l} \sum_{k=1}^{T_{r}} \alpha_{r k j} \delta_{r k}=0$ (Orthogonally conditions)

$\delta_{r k}>0$, (Positive constant)

\section{GEOMETRIC PROGRAMMING TECHNIQUE TO SOLVE FUZZY INVENTORY PROBLEM \& TESTING RESULTS}

The trapezoidal fuzzy number is $\bar{A}$ (a,b,c,d,) and the objective function is

$$
\overline{T(C(Q)}=\left[A+\sum_{i=1}^{n} C_{i} M_{i}+\sum_{i=1}^{n} d M_{i}+c Q+B+E+K+T+o\right] \frac{\bar{D}}{Q}+\frac{\bar{h} Q(1-x)}{2}
$$

Now, using ranking fuzzy numbers cost parameters, objective function becomes

$$
\begin{aligned}
& \overline{R_{\lambda, f}(T(C(Q))}= \\
& {\left[A+\sum_{i=1}^{n}\left(\bar{C}_{i}\right) M_{i}+\sum_{i=1}^{n} R_{\lambda, f}(\bar{d}) M_{i}+c Q+R_{\lambda, f}(B)+R_{\lambda, f}(E)+R_{\lambda, f}(K)+R_{\lambda, f}(T)+R_{\lambda, f}(o)\right]}
\end{aligned}
$$

Applying Geometric programming technique, we get, $\left.+\frac{R_{\lambda, f}(\bar{h})(1-x) Q}{2}\right\} w 1 r / Q w_{1 r}{ }^{*}\left\{\frac{Q(1-x) R_{\lambda, f}(h)}{2 w_{2 r}}\right\} w 2 r *\left(\frac{c R_{\lambda, f}(D)}{w_{3 r}}\right)^{w_{3 r}}$

\section{Subject to the conditions}

$\mathrm{W}_{1 \mathrm{r}}+\mathrm{W}_{2 \mathrm{r}}+\mathrm{W}_{3 \mathrm{r}}=1$

$-\mathrm{W}_{1 \mathrm{r}}+\mathrm{W}_{2 \mathrm{r}}=0$

$\mathrm{W}_{1 \mathrm{r}}+\mathrm{W}_{3 \mathrm{r}}=0$

Solving these conditions, we get the values of $\mathrm{W} 1 \mathrm{r}=\mathrm{W} 2 \mathrm{r}$ $=1$ and $\mathrm{W} 3 \mathrm{r}=-1$

By applying Duffin`s and peterson`s theorem,

$$
\begin{aligned}
& {\left[A+\sum_{i=1}^{n}\left(\bar{C}_{i}\right) M_{i}+\sum_{i=1}^{n} R_{\lambda, f}(\bar{d}) M_{i}+c Q+R_{\lambda, j}(B)+R_{\lambda, j}(E)+R_{\lambda, f}(K)+R_{\lambda, j}(T)+R_{\lambda, j}(o)\right] \frac{R_{\lambda, f}(\bar{D})}{Q} } \\
= & w_{1 r} g\left(w_{1 r}, w_{2 r}\right) \frac{R_{\lambda, f}(\bar{h})(1-x) Q}{2}=w_{2 r} g\left(w_{1 r}, w_{2 r}\right)
\end{aligned}
$$

From this, we obtain the optimal production quantity,

$$
\begin{aligned}
& Q^{*}=\left\{2 *\left[A+\sum_{i=1}^{n}\left(\bar{C}_{i}\right) M_{i}+\sum_{i=1}^{n} R_{\lambda, f}(\bar{d}) M_{i}+c Q+R_{\lambda, f}(B)+R_{\lambda, f}(E)+R_{\lambda, f}(K)\right] \frac{R_{\lambda, f}(\bar{D})}{Q},\right. \\
& \frac{\left.R_{\lambda, f}(\bar{h})(1-x)\right\}}{2} \cdots \ldots \ldots \ldots(2)
\end{aligned}
$$

\section{NUMERICAL EXAMPLE \& RESULTS}

Crisp Model:

The following parametric values are allowed in
$\prod_{i=1}^{n}=\left\{\left[A+\sum_{i=1}^{n}\left(\bar{C}_{i}\right) M_{i}+\sum_{i=1}^{n} R_{\lambda, f}(\bar{d}) M_{i}+c Q+R_{\lambda, j}(B)+R_{\lambda, j}(E)+R_{\lambda, j}(K)+R_{\lambda, j}(T)+R_{\lambda, j}(o)\right] \frac{R_{\lambda, f}(\bar{D})}{Q}\right.$

appropriate units:

$\mathrm{P}=150$ units/time, $\mathrm{A}=100 \varsigma, \mathrm{D}=100$ units/time, $\mathrm{B}=$ $250 \varsigma, \mathrm{d} \varsigma 15, \mathrm{~h}=\varsigma 0.5$ units/time, $\mathrm{k}=\varsigma 75, \mathrm{E}=\varsigma 50, \mathrm{C}_{1}=\varsigma 1.5, \mathrm{C}_{2}$ $=\varsigma 2 /$ unit $\mathrm{M}_{1}=15$ units, $\mathrm{T}=\varsigma 873, \mathrm{O}=\varsigma 300$.

The optimal solution is 1637 units.

Fuzzy Model: From (1), when $\lambda=0.6$

$$
\bar{D}=(96,98,102,104) R(\bar{D})=100.5 \sum_{i=1}^{n} R\left(C_{i}\right) M_{i}=
$$$$
(40,50,70,80)=62.6 \sum_{i=1}^{n} R(d) M_{i}=526.3
$$

$\bar{B}=(240,245,255,260) \quad R(\bar{B})=251 \quad \bar{E}=(48,49,51,52)$ $R(\bar{E})=50.6$

$$
\bar{K}=(73,74,76,77) R(\bar{K})=75.26 \bar{T}=(871,872,874,875)
$$

$R(\bar{T})=873.26$

$$
\bar{O}=(298,299,301,302) \quad R(\bar{O})=300.26 \bar{h}=
$$

$(0.3,0.4,0.6,0.7) \quad R(\bar{h})=0.52$

$$
\bar{P}=(148,149,151,152) \quad R(\bar{P})=150.1
$$

From (2)

$\mathrm{Q}^{*}=1617$ units.

\section{SENSITIVITY ANALYSIS:}

The affectability research is completed for checking the adequacy of the financial introduction amount version with condition cost effective adaptable and strong generation framework utilizing Geometric programming approach. This condition mirror the genuine desires of the creation framework and this assist the makers in handling each the internal and outdoor difficulties, client upkeep.

\section{CONCLUSION}

on this paper, a fluffy monetary generation amount version quantity model with circumstance supportable adaptable and strong of the creation framework utilising geometric programming strategy is gotten. The expenses are regarded as a trapezoidal fluffy quantity and after that these fluffy numbers are characterized by means of positioning fluffy quantity as for the high-quality wager meantime quantity . here, we've taken the estimation of $\cdot=0.6$ which ensures that the organisation can accomplish the idealistic stage. therefore the agency enables a few adaptability to perform his goal.

\section{REFERENCES}

1. DUFFIN, R. J., and PETERSON, E. L., 1974, limited minima treated by geometric techniques. Westinghouse Scienti c paper sixty four-158-129-P3

2. HARIRI, A. M. An., ABOU - EL-ATA,M. O., and pass YAL, S. k., 1995, The asset pressured multi-issue stock difficulty with value markdown: a geometric programming approach, generation arranging and control, 6, 374 • 377 .

three. HARIRI, A. M. An., ABOU - EL-ATA,M. O., 1995, Multi-aspect creation parcel length stock model with converting request cost 
under a confinement: a geometric programming method. Unpublished paper.

4. LEV, B., and WEISS, H. J., 1990 stock fashions with price changes, Operations studies, 38, 53-sixty three.

5. R. E. Bellman, L. A. Zadeh, choice making in a fluffy area, control $\begin{array}{llll}\text { science, } & 17 & \text { (1970) } & \text { B141-B164 }\end{array}$ https://doi.org/10.1287/mnsc.17.4.B141

6. B. Y. Cal, The further investigation of posynomial GP with fluffy co-e - cient, Mathematic Applicata, 5 (four) (1992) 119-a hundred and twenty

7. C. Carlsson, P. Korhonen, A parametric way to deal with fluffy direct programming, Fuzzy units and framework, (1986) 17-30. https://doi.org/10.1016/S0165-0114(86)80028-eight eight. A. J. Clark, a informal overview of multy-echelon inventory hypothesis, maritime research calculated Quarterly, 19 (2012) 621-650.

9. D. Dutta, Pavan Kumar, Fuzzy stock with out deficiencies using trapezoidal fluffy variety with affectability exam, IOSR journal of $\begin{array}{llll}\text { technology, four (three) 32-37. } & \text { (2012) }\end{array}$ https://doi.org/10.9790/5728-0433237

10. D. Dutta, J. R. Rao, R. N. Tiwary, impact of resilience in fluffy direct fragmentary programming, Fuzzy units and frameworks, 55 (1993) 133-142. https://doi.org/10.1016/0165-0114(ninety three) $90126-3$

11. Mandal, N. okay., Roy, T. okay. what's more, Maiti, M. Multi-objective fluffy inventory model with 3 barriers: a geometrical programming approach. Fluffy sets and structures. 2005. 150:87-106.

12. Liu, S. T. using geometric programming to gain growth with period in-between cofficients and quantity rebate. linked mathematics and Computation. 2009. 209: 259-265.

13. Leung, k. N. F. A summed up geometric programming solution for a financial advent amount version with adaptability and unwavering first-class contemplations. ecu diary of Operational research. 2007. 176: $240-251$.

14. Sadjadi, S. J., Ghazahfari, M. what's more, Yousefli, A. Fluffy valuing and marketing arranging model: A possibilistic geometric programming approach. master structures with packages. 2010. 37 3392-3397.

15. Mandal, N. okay., Fuzzy financial request quantity model with positioning fluffy wide variety price parameters. Yugoslav journal of Operations research. 2012. 22: 247-264.

16. Werners, B. sensible numerous goal programming difficulty to adaptable imperatives. ecu diary of Operational studies. 1987. 31: 342-349. 